\title{
Degeneracy and Colorings of Squares of Planar Graphs without 4-Cycles
}

\author{
Ilkyoo Choi* Daniel W. Cranston ${ }^{\dagger} \quad$ Théo Pierron ${ }^{\ddagger}$
}

September 25, 2019

\begin{abstract}
We prove several results on coloring squares of planar graphs without 4-cycles. First, we show that if $G$ is such a graph, then $G^{2}$ is $(\Delta(G)+72)$-degenerate. This implies an upper bound of $\Delta(G)+73$ on the chromatic number of $G^{2}$ as well as on several variants of the chromatic number such as the list-chromatic number, paint number, Alon-Tarsi number, and correspondence chromatic number. We also show that if $\Delta(G)$ is sufficiently large, then the upper bounds on each of these parameters of $G^{2}$ can all be lowered to $\Delta(G)+2$ (which is best possible). To complement these results, we show that 4-cycles are unique in having this property. Specifically, let $S$ be a finite list of positive integers, with $4 \notin S$. For each constant $C$, we construct a planar graph $G_{S, C}$ with no cycle with length in $S$, but for which $\chi\left(G_{S, C}^{2}\right)>\Delta\left(G_{S, C}\right)+C$.
\end{abstract}

\section{Introduction}

The square, $G^{2}$, of a graph $G$ is formed from $G$ by adding an edge $v w$ for each pair of square vertices, $v$ and $w$, at distance two in $G$. It is easy to check that $\chi\left(G^{2}\right) \leqslant \Delta\left(G^{2}\right)+1 \leqslant$ $\Delta(G)^{2}+1$, and this bound can be tight, as when $G$ is the 5-cycle or the Petersen graph (here $\chi$ and $\Delta$ denote, respectively, the chromatic number and maximum degree) 1 Even when $\Delta(G)$ is arbitrarily large, there exist constructions showing that this upper bound on $\chi(G)$ cannot be improved much. For example, when $G$ is the incidence graph

*Department of Mathematics, College of Natural Sciences, Hankuk University of Foreign Studies (HUFS), Republic of Korea; ilkyoo@hufs.ac.kr; Supported by the Basic Science Research Program through the National Research Foundation of Korea (NRF) funded by the Ministry of Education (NRF2018R1D1A1B07043049), and also by the Hankuk University of Foreign Studies Research Fund.

${ }^{\dagger}$ Department of Mathematics and Applied Mathematics, Virginia Commonwealth University, Richmond, VA, USA; dcranston@vcu.edu; This research is partially supported by NSA Grant H98230-15-1-0013.

${ }^{\ddagger}$ Univ. Bordeaux, Bordeaux INP, CNRS, LaBRI, UMR 5800, F-33400 Talence, France; tpierron@labri.fr

${ }^{1}$ For simplicity, in this introduction we discuss only standard vertex coloring. But starting in Section 2 we consider degeneracy, and at the end of that section we mention multiple other graph coloring parameters. 
of a projective plane $2^{2}$ we have $\chi\left(G^{2}\right) \approx \Delta(G)^{2}-\Delta(G)$. However, for planar graphs, we have much better bounds on $\chi\left(G^{2}\right)$.

Recall that Euler's formula implies that every planar graph $G$ is 5 -degenerate. Coloring vertices greedily in the reverse of this degeneracy order [10], [6, Theorem 4.9] shows that $\chi\left(G^{2}\right) \leqslant 9 \Delta(G)$. Refinements of this approach have led to successive improvements of this upper bound, culminating with the result of Molloy and Salavatipour [11] that $\chi\left(G^{2}\right) \leqslant\left\lceil\frac{5}{3} \Delta(G)\right\rceil+78$. Havet et al. [9] also proved that $\chi\left(G^{2}\right) \leqslant \frac{3}{2} \Delta(G)(1+o(1))$, which strengthens the bound of [11] when $\Delta(G)$ is sufficiently large. Amini et al. [2] proved the same bound for all graphs embeddable in any fixed surface.

Every graph $G$ satisfies $\chi\left(G^{2}\right) \geqslant \Delta(G)+1$, and for planar graphs we might naively hope to prove a matching upper bound, or at least a bound of the form $\chi\left(G^{2}\right) \leqslant$ $\Delta(G)+C$, for some constant $C$. However, for each $k \in \mathbb{Z}^{+}$, Wegner constructed a planar graph $G_{k}$ with $\Delta\left(G_{k}\right)=k$ and $\chi\left(G_{k}^{2}\right)=\left\lfloor\frac{3}{2} k\right\rfloor+1$; Figure 1 shows his construction. So to prove a bound of the form $\chi\left(G^{2}\right) \leqslant \Delta(G)+C$, we must restrict to some proper subset of planar graphs.

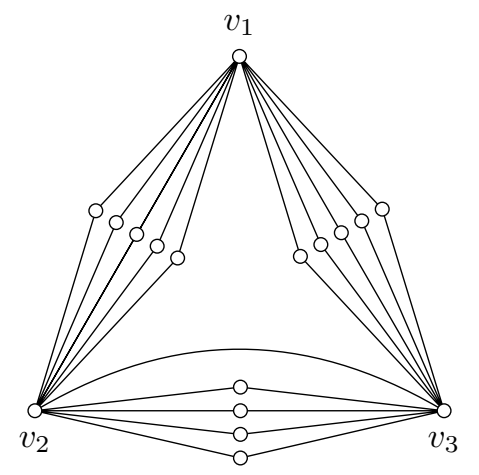

Figure 1: Wegner's construction

Wang and Lih 13 . conjectured that, for each $g \geqslant 5$, there exists $D_{g}$ such that if $G$ is a planar graph with girth at least $g$ and $\Delta(G) \geqslant D_{g}$, then $\chi\left(G^{2}\right)=\Delta(G)+1$. This is true for $g \geqslant 7[4$. But it is false for girth 5 and 6 since, for each $k \geqslant 3$, there exists a planar graph $G_{k}$ with $\Delta\left(G_{k}\right)=k$ and with girth 6 such that $\chi\left(G_{k}^{2}\right)=\Delta\left(G_{k}\right)+2$ [4]. However, Dvořák et al. [8] proved a surprising complementary result: $\chi\left(G^{2}\right) \leqslant \Delta(G)+2$ whenever $G$ is a planar graph with girth 6 and $\Delta(G)$ sufficiently large. This work inspired analogous results for planar graphs with (i) girth 5 [3] and (ii) no 4-cycles or 5 -cycles (though 3 -cycles are allowed) $[7]^{3}$. In each case the bound $\chi\left(G^{2}\right) \leqslant \Delta(G)+2$ still holds (though the required lower bound on $\Delta(G)$ is larger).

The work above naturally leads to the following question. Exactly which cycle lengths can be forbidden from planar graphs to get a bound of the form $\chi\left(G^{2}\right) \leqslant$ $\Delta(G)+C$ ? For a set $\mathcal{S}$ of positive integers, let $\mathcal{G}_{\mathcal{S}}$ denote the family of planar graphs having no cycles with length in $\mathcal{S}$.

\footnotetext{
${ }^{2}$ This incidence graph $G$ is $(k+1)$-regular and bipartite with each part of size $k^{2}+k+1$. Since each pair of vertices within a part has a common neighbor, $\omega\left(G^{2}\right)=k^{2}+k+1$.

${ }^{3}$ Here we only hit the highlights. For a more detailed history of this problem, we recommend the introduction of [9] and [6, Conjecture $4.7 \mathrm{ff}$.$] .$
} 
Main Theorem. For a finite set $\mathcal{S}$ there exists a constant $C_{\mathcal{S}}$ such that $\chi\left(G^{2}\right) \leqslant$ $\Delta(G)+C_{\mathcal{S}}$ for all $G \in \mathcal{G}_{\mathcal{S}}$ if and only if $4 \in \mathcal{S}$.

We prove the Main Theorem in two parts. Immediately below we give a construction that proves the "only if" part. In Section 2 we handle the "if" part, the case when $4 \in \mathcal{S}$. In fact, we prove the stronger statement that the vertices of every graph $G \in \mathcal{G}_{\{4\}}$ can be ordered so that each vertex is preceded in the order by at most $\Delta(G)+72$ of its neighbors in $G^{2}$. Now the coloring result follows by coloring greedily. In Section 3 , when $\Delta(G)$ is sufficiently large we strengthen our bound to $\chi\left(G^{2}\right) \leqslant \Delta(G)+2$, which is sharp. This bound also holds for paint number, Alon-Tarsi number, and correspondence chromatic number (all defined at the end of Section 2).

Lemma 1.1. If $4,2 k \notin \mathcal{S}$, for some odd integer $k \geqslant 3$, then there does not exist a constant $C_{\mathcal{S}}$ such that $\chi\left(G^{2}\right) \leqslant \Delta(G)+C_{\mathcal{S}}$ for every $G \in \mathcal{G}_{\mathcal{S}}$.

Proof. Begin with a $k$-cycle and replace each edge $v w$ with a copy of $K_{2, t}$, so that the two vertices of degree $t$ replace $v$ and $w$. The resulting graph, $G_{k, t}$ has maximum degree $2 t$ and has cycles only of lengths 4 and $2 k$. In every proper coloring of $G_{k, t}^{2}$, each color class contains at most $(k-1) / 2$ vertices of degree 2 in $G_{k, t}$ (by the Pigeonhole Principle). Since $G_{k, t}$ has $k t$ vertices of degree 2 , we get $\chi\left(G_{k, t}^{2}\right) \geqslant k t /((k-1) / 2)=$ $2 k t /(k-1)=2 t+2 t /(k-1)=\Delta(G)+2 t /(k-1)$. Given any constant $C$, we can choose $t$ sufficiently large so that $2 t /(k-1)>C$.

\section{Graphs with no 4-cycles}

Our goal in this section is to prove Theorem 2.1, below. First we need a few definitions. A $k$-vertex (resp. $k^{+}$-vertex, $k^{-}$-vertex) is a vertex of degree equal to (resp. at least, at most) $k$; a $k$-neighbor, of a vertex $v$, is an adjacent $k$-vertex. Analogously, we define $k$-face, $k^{+}$-face, and $k^{-}$-face. We write $d(v)$ for the degree of a vertex $v$ and $\ell(f)$ for the length of a face $f$. We write $N[v]$ to denote $N(v) \cup\{v\}$ and $N[S]$ for $\cup_{v \in S} N[v]$. We write $N^{2}(v)$ for the set of neighbors of $v$ in $G^{2}$. When the context could be unclear, we specify our meaning by using $d_{G}, N_{G}$, and $N_{G}^{2}$. An order, $\sigma$, of $V(G)$ is $\operatorname{good}$ for $G$ if each vertex, $v$, of $G$ is preceded in $\sigma$ by at most $\Delta(G)+72$ vertices in $N^{2}(v)$. Following the approach of [5], we prove the degeneracy result below, which immediately implies the desired coloring bounds, by coloring greedily.

Theorem 2.1. For every planar graph $G$ with no 4-cycles, there exists a vertex order $\sigma$ such that each vertex $v$ is preceded in $\sigma$ by at most $\Delta(G)+72$ of its neighbors in $G^{2}$.

Our proof of Theorem 2.1 is by discharging, with initial charge $d(v)-4$ for each vertex $v$ and $\ell(f)-4$ for each face $f$. In the next section we discuss the discharging rules, but for now it is enough to note that we only need to give extra charge to 2-vertices, 3 -vertices, and 3 -faces. Here we prove that certain configurations are reducible; that is, they cannot appear in a minimal counterexample. In each case we assume that our minimal counterexample $G$ contains such a configuration. We modify $G$ to get a smaller graph $G^{\prime}$ (that is also planar and without 4-cycles), and which therefore has the desired vertex order, $\sigma^{\prime}$. Finally, we modify $\sigma^{\prime}$ to get $\sigma$, a good vertex order for

$k$-vertex

$k$-neighbor

$k$-face

$N[v], N[S], N^{2}(v)$ good for $G$ 
$G$ of $V(G)$. Each reducible configuration formalizes the intuition that every 2-vertex, 3 -vertex, and 3-face of $G$ must be near a vertex $v$ of high degree. This is useful, since $v$ has extra charge to share with nearby vertices and faces that need it.

Proof of Theorem 2.1. Suppose the theorem is false, and let $G$ be a counterexample that minimizes the number of $3^{+}$-vertices and, subject to that, the number of edges. If $G$ is disconnected, then we can get a good vertex order for each component (by minimality) and concatenate these to get a good order for $G$. Thus, $G$ is connected. Similarly, if $G$ has a 1-vertex $v$, then $G-v$ has a good order $\sigma^{\prime}$ and we can append $v$ to $\sigma^{\prime}$. So $G$ has no 1 -vertex. A vertex $v$ is big if $d(v) \geqslant 10$, and $v$ is small if $5 \leqslant d(v) \leqslant 9$. Note that $\Delta(G) \geqslant 10$, since otherwise each vertex has at most $9^{2}$ neighbors in $G^{2}$, so every vertex order shows that $G$ is not a counterexample.

\subsection{Reducible Configurations}

Key Lemma. For an edge vw in $G$, if both $v$ and $w$ are not big, then at least one of $v$ and $w$ has at least two big neighbors.

Proof. Suppose to the contrary that both $v$ and $w$ are not big, and that each has at most one big neighbor. By minimality, $G-v w$ has a good order, $\sigma^{\prime}$. By deleting $v$ and $w$ from $\sigma^{\prime}$, we get a good order (for $G$ ) of $V(G)-\{v, w\}$. Since $v$ is not big and has at most one big neighbor, $\left|N^{2}(v)\right| \leqslant \Delta(G)+(10-1)(10-2)$. By symmetry, $\left|N^{2}(w)\right| \leqslant \Delta(G)+(10-1)(10-2)$. Thus, by appending $v$ and $w$ to the order, we get a good order for $G$, which is a contradiction.

Lemma 2.2. If a 3-face $f$ is incident with a 2-vertex, then the other two vertices on $f$ must be big vertices.

Proof. Let $v w_{1} w_{2}$ be a 3 -face that is incident with a 2-vertex $v$. Suppose to the contrary that $w_{1}$ is not big. By minimality, $G-v$ has a good order, which is a good order for $G$ of $V(G)-\{v\}$. Since $w_{1}$ is not big, $\left|N^{2}(v)\right| \leqslant \Delta(G)+7$. Thus, we can append $v$ to obtain a good order of $G$, which is a contradiction.

Lemma 2.3. Every 3-face that is incident with two 3-vertices is also incident with a big vertex.

Proof. Suppose that a 3 -face is incident with two 3 -vertices $v_{1}, v_{2}$ and a vertex $w$. Applying the Key Lemma to $v_{1} v_{2}$ shows that $w$ must be big.

Lemma 2.4. Every 3-vertex has a big neighbor.

Proof. Let $v$ be a 3 -vertex with neighbors $w_{1}, w_{2}, w_{3}$. Suppose to the contrary that every $w_{i}$ is not big. Applying the Key Lemma to each edge $v w_{i}$ shows that each $w_{i}$ must be a $3^{+}$-vertex. Consider the graph $G^{\prime}$ formed from $G-v$ by adding a path of length two between each pair of neighbors of $v$. (Since each $w_{i}$ is not big, we have $\Delta\left(G^{\prime}\right)=\Delta(G)$.) Since $G^{\prime}$ has fewer $3^{+}$-vertices, by minimality $G^{\prime}$ has a good order $\sigma^{\prime}$, and $\sigma^{\prime}$ also is a good order for $G$ of $V(G)-v$. Since each neighbor of $v$ is small, $\left|N^{2}(v)\right| \leqslant 3 \cdot 9$. So appending $v$ to $\sigma^{\prime}$ gives a good order for $G$, which is a contradiction. 
Lemma 2.5. If a 3-face $f$ is incident with a 3-vertex $v$ and at most one big vertex, then the neighbor of $v$ that is not on $f$ must be a big vertex.

Proof. Let $v$ be a 3 -vertex on a 3 -face $v w_{1} w_{2}$ and let $x$ be the neighbor of $v$ that is not on $v w_{1} w_{2}$. Suppose to the contrary that both $w_{1}$ and $x$ are not big. Applying the Key Lemma to edge $v x$ shows that $x$ is a $3^{+}$-vertex. Consider the graph $G^{\prime}$ formed from $G-v$ by adding paths of length two between $x$ and $w_{1}$ and also between $x$ and $w_{2}$. So, $G^{\prime}$ has fewer $3^{+}$-vertices than $G$. By minimality, $G^{\prime}$ has a good order, $\sigma^{\prime}$, which also is a good order for $G$ of $V(G)-v$. Since $v$ has at most one big neighbor, $\left|N^{2}(v)\right| \leqslant \Delta(G)+16$. So appending $v$ to $\sigma^{\prime}$ gives a good order for $G$, which is a contradiction.

\subsection{Discharging}

We use the initial charges $d(v)-4$ for each vertex $v$ and $\ell(f)-4$ for each face $f$. Note that, by Euler's formula, the sum of these initial charges is -8 . Using the structural lemmas in Section 2.1, we redistribute this charge so that each vertex and face ends with nonnegative charge. However, this gives a contradiction, since a sum of nonnegative numbers cannot equal -8 . To redistribute charge, we use the following six discharging rules, applied in succession. (See Figure 2 for an illustration of the discharging rules.)

(R1) Each edge takes $\frac{1}{5}$ from each incident $5^{+}$-face and $\frac{1}{10}$ from each incident big vertex

(R2) If edge $v w$ is incident to a 3 -face $f$, then $v w$ gives all its charge (received by $(\mathrm{R} 1))$ to $f$. Otherwise, $v w$ distributes its charge equally among incident vertices $x$ where $d(x)=\min \{d(v), d(w)\}$.

(R3) Each big vertex gives $\frac{1}{2}$ to each neighbor.

(R4) Each 3-vertex, 4-vertex, and small vertex gives $\frac{3}{5}$ to each 2-neighbor. If either $v$ is a 4 -vertex with at least two big neighbors or $v$ is a small vertex, then $v$ gives $\frac{1}{2}$ to each incident 3 -face that is incident with a vertex other than $v$ that is not big.

(R5) Assume vertices $v$ and $w$ are big and the edge $v w$ lies on a 3-face $v w x$. If $x$ is a $4^{-}$-vertex, then all charge given from $v$ to $w$ (and vice versa) by (R3) continues on to $x$. If $x$ is a $5^{+}$-vertex, then all charge given from $v$ to $w$ (and vice versa) by (R3) continues on to face $v w x$.

(R6) If a 3 -vertex has an incident 3-face $f$ with negative charge, then $v$ gives its excess charge to $f$.

Now we show that each vertex and face ends with nonnegative charge, which yields the desired contradiction.

Each $5^{+}$-face $f$ ends with charge $\ell(f)-4-\frac{1}{5} \ell(f)=\frac{4}{5} \ell(f)-4 \geqslant 0$. Each edge receives charge by (R1) and gives it all away by (R2), so ends with 0 . Consider a big vertex $v$. For each of its neighbors $w$, the charge that $v$ gives to $v w$ by (R1) is $\frac{1}{10}$ and to $w$ by (R3) is $\frac{1}{2}$, for a total of $\frac{3}{5}$. So $v$ ends with at least $d(v)-4-\frac{3}{5} d(v)=\frac{2}{5} d(v)-4$; this is nonnegative, since $d(v) \geqslant 10$.

\footnotetext{
${ }^{4}$ A cut-edge takes $\frac{2}{5}$ from its incident face.
} 


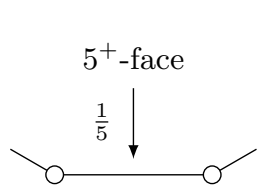

(R1)

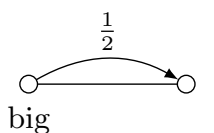

(R3)

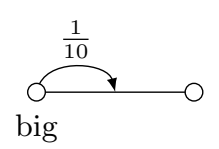

(R1)

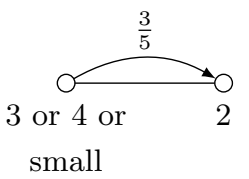

(R4)

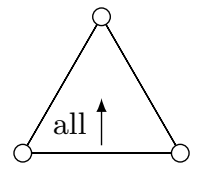

(R2)

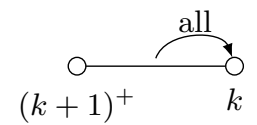

(R2)

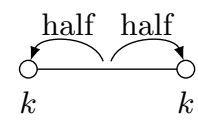

(R2)

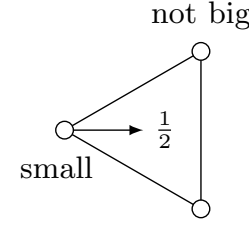

(R4)

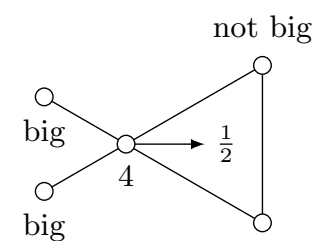

(R4)

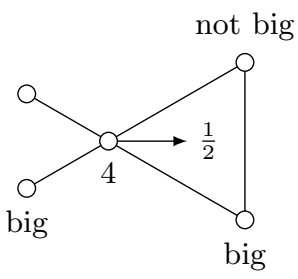

(R4)

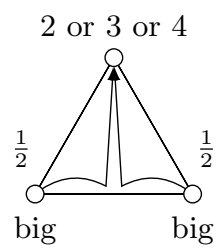

(R5)

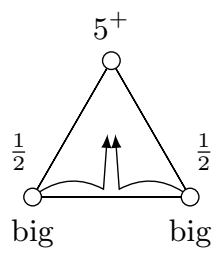

(R5)

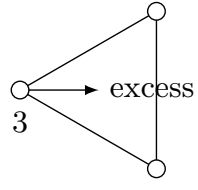

(R6)

Figure 2: An illustration of the discharging rules.

Consider a small vertex $v$. Let $n_{2}(v)$ denote the number of 2-neighbors of $v$ and $f_{3}(v)$ the number of 3 -faces incident with $v$ that are not incident with two big neighbors of $v$ (that is, 3 -faces that get $\frac{1}{2}$ from $v$ ). By (R4), $v$ gives away $\frac{3}{5} n_{2}(v)+\frac{1}{2} f_{3}(v)$. Let $w_{1}, \ldots, w_{d(v)}$ denote the neighbors of $v$. Suppose that $v$ has a 2-neighbor. By the Key Lemma, $v$ has at least two big neighbors, so $n_{2}(v) \leqslant d(v)-2$. By Lemma 2.2 , since $v$ is small, if $w_{i}$ is a 2-vertex, then neither face incident with $v w_{i}$ receives charge from $v$. Thus, $n_{2}(v)+f_{3}(v) \leqslant d(v)$. By a more careful analysis, we will show that $n_{2}(v)+f_{3}(v) \leqslant d(v)-2$. If $f_{3}(v)=0$, then this inequality follows from $n_{2}(v) \leqslant d(v)-2$ above. So assume $f_{3}(v) \geqslant 1$ and that $v$ sends charge to some 3 -face $f^{\prime \prime}$ with boundary that includes $w_{2} v w_{3}$. Since $G$ has no 4 -cycles, neither the face preceding $f^{\prime \prime}$ around $v$ nor the face following $f^{\prime \prime}$ is a 3 -face, so neither of them receives charge from $v$; call these faces $f^{\prime}$ and $f^{\prime \prime \prime}$. Further, some $w_{j}$ other than $w_{2}$ and $w_{3}$ is big. Thus, we can pair the neighbors of $v$ other than $w_{2}, w_{3}$, and $w_{j}$ with the faces other than $f^{\prime}, f^{\prime \prime}$, and $f^{\prime \prime \prime}$ such that each face is paired with an incident vertex and at most one element in each pair gets charge from $v$. This proves $n_{2}(v)+f_{3}(v) \leqslant d(v)-2$. So, $v$ ends with at least $d(v)-4+2\left(\frac{1}{2}\right)-\frac{3}{5}(d(v)-2)=\frac{2}{5} d(v)-\frac{9}{5}$; this is positive, since $d(v) \geqslant 5$. Now instead assume that $v$ has no 2-neighbors. Since $G$ has no 4-cycles, $f_{3}(v) \leqslant\left\lfloor\frac{d(v)}{2}\right\rfloor$. 
Thus, $v$ ends with at least $d(v)-4-\frac{1}{2}\left\lfloor\frac{d(v)}{2}\right\rfloor$; this is nonnegative since $d(v) \geqslant 5$.

So, to complete the proof we only need to consider 3 -faces, 2-vertices, 3-vertices, and 4 -vertices.

Claim 2.6. Every 2-vertex $v$ that is on a 3 -face vw $w_{1} w_{2}$ ends with nonnegative charge.

Proof. By Lemma 2.2 , both $w_{1}$ and $w_{2}$ must be big. By (R3), $v$ gets $\frac{1}{2}$ from each of $w_{1}$ and $w_{2}$. And by (R5), $v$ gets another $2\left(\frac{1}{2}\right)$. So $v$ ends with $2-4+4\left(\frac{1}{2}\right)=0$.

Claim 2.7. Every 2-vertex $v$ that is not on a 3-face ends with nonnegative charge.

Proof. Let $w_{1}$ and $w_{2}$ be the neighbors of a 2-vertex $v$. It suffices to show that $v$ gets total charge at least 1 from $w_{1}$ and $v w_{1}$, since by symmetry it also gets at least 1 from $w_{2}$ and $v w_{2}$, so $v$ ends with at least $2-4+2(1)=0$. Applying the Key Lemma to $v w_{1}$ shows that $w_{1}$ either is big or is a $3^{+}$-vertex with two big neighbors. By (R1), vw $w_{1}$ gets $\frac{2}{5}$ from incident faces and by (R2) $v w_{1}$ gives all this charge to $v$. So we only need to show that $v$ gets at least $\frac{3}{5}$ from $w_{1}$. If $w_{1}$ is a $3^{+}$-vertex that is not big, then $w_{1}$ gives $\frac{3}{5}$ to $v$ by (R4). If $w_{1}$ is big, then it gives $v$ charge $\frac{1}{2}$ by (R3), and gives edge $v w_{1}$ an extra $\frac{1}{10}$ by (R1), and all this charge goes to $v$ by (R2). Thus, $v$ gets $\frac{3}{5}$, as desired.

Claim 2.8. Every 3-vertex $v$ ends with nonnegative charge.

Proof. By Lemma 2.4, $v$ has a big neighbor $w$.

First suppose that $v$ does not have a 2-neighbor. If $v w$ is not on a 3 -face, then by (R3) $w$ gives $v$ charge $\frac{1}{2}$, and by (R2) edge $v w$ gives $v$ charge $\frac{2}{5}+\frac{1}{10}$. So $v$ ends with at least $3-4+\frac{1}{2}+\frac{2}{5}+\frac{1}{10}=0$. So assume $v$ is on a 3 -face and $v z$ is on a 3 -face for every big neighbor $z$ of $v$. By Lemma 2.5, vertex $v$ has at least two big neighbors, say $w_{1}$ and $w_{2}$. Since each of $v w_{1}$ and $v w_{2}$ must be on a 3-face, and $v$ has only a single incident 3 -face, it must be $v w_{1} w_{2}$. Now, by (R3) and (R5), $v$ gets at least $4\left(\frac{1}{2}\right)$. So $v$ ends (R5) with at least $3-4+4\left(\frac{1}{2}\right)>0$.

Now assume that $v$ has a 2-neighbor $x$, which gets $\frac{3}{5}$ from $v$ by (R4). Since $d(v)=3$ and $d(x)=2$, Lemma 2.2 implies that $v x$ is not incident to any 3-face. Applying the Key Lemma to $v x$ shows that $v$ has two big neighbors, $w_{1}$ and $w_{2}$. If $v w_{1} w_{2}$ is a 3 -face, then each of $w_{1}$ and $w_{2}$ gives $\frac{1}{2}+\frac{1}{2}$ to $v$, by (R3) and (R5). So $v$ ends with at least $3-4-\frac{3}{5}+4\left(\frac{1}{2}\right)>0$. If $v w_{1} w_{2}$ is not a 3 -face, then each of $v w_{1}$ and $v w_{2}$ gives $\frac{1}{2}$ to $v$, by (R1) and (R2). So $v$ ends with at least $3-4-\frac{3}{5}+2\left(\frac{1}{2}\right)+2\left(\frac{1}{2}\right)>0$.

Claim 2.9. Every 4-vertex $v$ ends with nonnegative charge.

Proof. Let $n_{2}(v)$ and $f_{3}(v)$ denote, respectively, the numbers of 2-neighbors and incident 3 -faces that get charge from $v$ by (R4).

Suppose $v$ has no 2-neighbor. If $v$ gives no charge to incident 3 -faces by (R4), then $v$ gives no charge at all, so $v$ ends with at least $4-4=0$. If $v$ does give charge to an incident 3-face by (R4), then (R4) implies that $v$ has two big neighbors; by (R3), each big neighbor gives $v$ charge $\frac{1}{2}$. Since $G$ has no 4 -cycles, $v$ gives charge to at most two 3 -faces. So $v$ ends with at least $4-4+2\left(\frac{1}{2}\right)-2\left(\frac{1}{2}\right)=0$.

So assume $v$ has a 2-neighbor, $u$. Applying the Key Lemma to $u v$ shows that $v$ has two big neighbors, $w_{1}$ and $w_{2}$; by (R3) each $w_{i}$ gives $v$ charge $\frac{1}{2}$. If $v w_{i}$ is not on a 
3 -face, for some $w_{i}$, then by (R2) $v w_{i}$ gives $v$ charge $\frac{2}{5}+\frac{1}{10}$. Thus, $v$ ends with at least $4-4+2\left(\frac{1}{2}\right)+\left(\frac{2}{5}+\frac{1}{10}\right)-2 \cdot \frac{3}{5}>0$. So we assume that each $v w_{i}$ is on a 3 -face. Since $v$ has a 2-neighbor (which is not on a 3 -face with $v$, by Lemma 2.2), and $G$ has no 4 -cycles, $v$ has at most one incident 3-face. Since $v w_{1}$ and $v w_{2}$ are both on 3 -faces, the 3 -face must be $v w_{1} w_{2}$. Because $w_{1}$ and $w_{2}$ are both big, $v$ gives no charge to $v w_{1} w_{2}$. So $v$ ends with at least $4-4+4\left(\frac{1}{2}\right)-2\left(\frac{3}{5}\right)>0$.

Claim 2.10. Every 3 -face ends with nonnegative charge.

Proof. Let $f=v_{1} v_{2} v_{3}$ be a 3 -face, where $d\left(v_{1}\right) \leqslant d\left(v_{2}\right) \leqslant d\left(v_{3}\right)$. By (R1) each of $v_{1} v_{2}, v_{2} v_{3}, v_{3} v_{1}$ gets $\frac{1}{5}$ from its incident $5^{+}$-face, and by (R2) all of this charge goes to $f$. If $f$ has two incident big vertices, then by (R1) edges $v_{1} v_{2}, v_{2} v_{3}, v_{3} v_{1}$ get in total an additional $\frac{4}{10}$. So $f$ ends with at least $3-4+\frac{3}{5}+\frac{4}{10}=0$. If $v_{1}$ is a 2 -vertex, then $v_{2}$ and $v_{3}$ are both big, by Lemma 2.2, and we are done, as above. So assume that $v_{1}$ is a $3^{+}$-vertex, and $v_{2}$ is not big. If some $v_{i}$ is a small vertex or a 4 -vertex with two big neighbors (which, by assumption, are not both incident to $f$ ), then $v_{i}$ gives $\frac{1}{2}$ to $f$ by (R4), so $f$ ends with at least $3-4+3\left(\frac{1}{5}\right)+\frac{1}{2}>0$. So we assume that $f$ has at most one incident big vertex, and has no incident small vertex, and no incident 4 -vertex with two big neighbors. Applying the Key Lemma to $v_{1} v_{2}$ shows that $f$ must have an incident big vertex. Otherwise $v_{1}$ and $v_{2}$ are each $4^{-}$-vertices with at most one big neighbor, a contradiction. Thus, we can assume that $f$ has exactly one incident big vertex, and has no incident 2-vertex, small vertex, or 4-vertex with two big neighbors.

So assume that $v_{3}$ is big and that $v_{1}$ and $v_{2}$ are each either a 3 -vertex or else a 4 -vertex with no big neighbor other than $v_{3}$. Applying the Key Lemma to $v_{1} v_{2}$ shows that $v_{1}$ must be a 3 -vertex. Furthermore, at least one of $v_{1}$ and $v_{2}$ is a 3 -vertex with a big neighbor $w$ not on $f$; by symmetry, assume this is $v_{1}$. By (R3), $w$ and $v_{3}$ each give $v_{1}$ charge $\frac{1}{2}$. Since edge $w v_{1}$ is not on a 3 -face, by (R2) it gives $v_{1}$ charge $\frac{2}{5}+\frac{1}{10}$. So $v_{1}$ finishes (R5) with at least $3-4+2\left(\frac{1}{2}\right)+\frac{2}{5}+\frac{1}{10}=\frac{1}{2}$; by (R6) all of this charge continues on to $f$. So $f$ ends with at least $3-4+3\left(\frac{1}{5}\right)+\frac{1}{2}>0$.

This completes the proof of Theorem 2.1.

For completeness, we conclude this section with the definitions of Alon-Tarsi number, paint number and correspondence chromatic number, and the corollary that bounds these parameters for planar graphs with no 4-cycles. To denote the listchromatic number of a graph $G$, we write $\chi_{\ell}(G)$.

An eulerian digraph is one in which each vertex has indegree equal to outdegree. For a digraph $D$, let $E E(D)$ and $E O(D)$ denote the numbers of eulerian subgraphs of $D$ in which the number of edges is even and odd, respectively. A digraph $D$ is AlonTarsi if $E E(D) \neq E O(D)$, and it is $k$-Alon-Tarsi if also each vertex has outdegree less than $k$. An orientation of a graph $G$ is formed from $G$ by directing each edge toward one of its endpoints. The Alon-Tarsi number of $G$, denoted $\operatorname{AT}(G)$, is the smallest $k$ such that some orientation of $G$ is $k$-Alon-Tarsi. Note that every acyclic orientation $D$ is Alon-Tarsi, since $E E(D)=1 \neq 0=E O(D)$; the only eulerian subgraph of $D$ is the spanning edgeless graph. Suppose that $G$ has degeneracy $k$, and $\sigma$ is a vertex ordering witnessing this. By orienting each edge toward its endpoint that appears earlier in $\sigma$, we conclude that $\operatorname{AT}(G) \leqslant k+1$. 
The paint number is defined using a two-player game. At round $i$, one player (Lister) chooses a set $S_{i}$ of vertices and the other one (Painter) answers by coloring an independent subset of $S_{i}$ with color $i$. The winning conditions depend on a fixed integer $k$ : Lister wins if he presents a vertex on $k$ rounds but Painter never colors it. Otherwise, Painter wins. The paint number $\chi_{p}(G)$ is the smallest integer $k$ such that Painter has a winning strategy with parameter $k$. This problem can be seen as a generalization of list coloring, where the lists are not all known at the beginning of the coloring process (take $S_{i}$ as the set of vertices whose lists contain color $i$ ). As shown by Schauz [12], each $k$-Alon-Tarsi graph is $k$-paintable. Thus, every $k$-degenerate graph $G$ satisfies $\chi_{p}(G) \leqslant \operatorname{AT}(G) \leqslant k+1$.

Given a graph $G$ and a function $f: V(G) \rightarrow \mathbb{N}$, an $f$-correspondence assignment $C$ is given by a matching $C_{v w}$, for each $v w \in E(G)$, between $\{v\} \times\{1, \ldots, f(v)\}$ and $\{w\} \times\{1, \ldots, f(w)\}$. We say that each vertex $x$ has $f(x)$ available colors. A $k$-correspondence assignment is an $f$-correspondence assignment where $f(v)=k$ for all $v \in V(G)$. Given an $f$-correspondence assignment $C$, a $C$-coloring is a function $\varphi: V(G) \rightarrow \mathbb{N}$ such that $\varphi(v) \leqslant f(v)$ for each $v \in V(G)$, and, for each edge $v w \in$ $E(G)$, the pairs $(v, \varphi(v))$ and $(w, \varphi(w))$ are nonadjacent in $C_{v w}$. The correspondence chromatic number of $G$, denoted $\chi_{\text {corr }}(G)$, is the least integer $k$ such that, for every $k$-correspondence assignment $C$ of $G$, graph $G$ admits a $C$-coloring. Note that if $G$ is $k$-degenerate, then coloring greedily in some order witnessing this shows that $\chi_{\text {corr }}(G) \leqslant k+1$. Thus, we have the following corollary of Theorem 2.1.

Corollary 2.11. If $G$ is a planar graph with no 4-cycles, then $\chi_{\text {corr }}\left(G^{2}\right) \leqslant \Delta(G)+73$, $\chi_{p}\left(G^{2}\right) \leqslant \Delta(G)+73$, and $A T\left(G^{2}\right) \leqslant \Delta(G)+73$.

\section{Graphs with no 4-cycles and $\Delta$ large}

In this section we show that the upper bounds in Corollary 2.11 can be strengthened to $\Delta(G)+2$ when $\Delta(G)$ is sufficiently large. Initially, we just prove this upper bound for $\operatorname{AT}\left(G^{2}\right)$, which also implies it for $\chi_{p}\left(G^{2}\right)$ and $\chi_{\ell}\left(G^{2}\right)$. In Section 3.6 we extend this result to $\chi_{\text {corr }}\left(G^{2}\right)$.

Theorem 3.1. There exists $\Delta_{0}$ such that if $G$ is a plane graph with no 4-cycles and with $\Delta(G) \geqslant \Delta_{0}$, then $G^{2}$ is $(\Delta(G)+2)$-choosable. In fact, $\chi_{p}\left(G^{2}\right) \leqslant A T\left(G^{2}\right) \leqslant \Delta(G)+2$.

Let $\Delta_{0}=23769500^{2}=564989130250000$ and fix $k \geqslant \Delta_{0}$. We prove by contradiction that if $G$ is a plane graph with no 4-cycles and with $\Delta(G) \leqslant k$, then $G^{2}$ is $(k+2)$-choosable. (By plane graph, we mean a planar graph with a fixed embedding in the plane. In particular, the neighborhood of each vertex is naturally endowed with a cyclic ordering.) For ease of exposition, we present the proof only for choosability, although it also works for paintability and Alon-Tarsi orientations. Most of the reducible configurations rely only on degeneracy, though at one point we use the kernel lemma.

Assume the theorem is false and let $G$ be a counterexample that minimizes $|E(G)|+$ $|V(G)|$. Let $L$ be an assignment of lists of size $k+2$ to the vertices of $G$ such that $G^{2}$ has no $L$-coloring. Throughout Section 3 we prove several structural lemmas, which 
ultimately lead to a contradiction. We follow the same general approach as in [3], which considered planar graphs with girth at least 5 ; however, we need new ideas to handle the presence of triangles.

\subsection{First Reducible Configurations}

Lemma 3.2. The graph $G$ is connected and has minimum degree at least 2 .

Proof. Note that $G$ is connected, since otherwise one of its components is a smaller counterexample. Now assume there exists a 1-vertex $v \in V(G)$. By the minimality of $G$, we can $L$-color $(G \backslash\{v\})^{2}$. Since $|L(v)|=k+2$, and $v$ has at most $1+(k-1)$ neighbors in $G^{2}$, we can color $v$ with a color not used on its neighbors in $G^{2}$, which is a contradiction.

The next two lemmas essentially show that every vertex of $G$ must be near a vertex of high degree. To formalize this, we use the following terminology: a vertex $v \in V(G)$ is big if $d(v) \geqslant \sqrt{k}$ and small otherwise. Denote by $B$ and $S$ the sets of big and small vertices. To refine the set $S$, we write $S_{i}$ for the set of small vertices with exactly $i$ big neighbors.

Remark 3.3. In our figures in the rest of the paper, we draw small vertices as circles, and big vertices as squares. Further, we use black circles for vertices with all neighbors shown. So a white vertex could have more neighbors than those shown; in fact, it could also have edges (that are not drawn) to other vertices that are shown. For example, Figure 3 shows the configurations forbidden by Lemma 3.4.

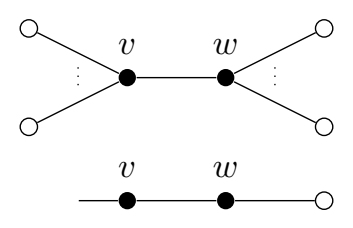

Figure 3: Forbidden configurations of Lemma 3.4

Lemma 3.4. For each edge $v w \in E(G)$, either $v \in N[B]$ or $w \in N[B]$. Further, if $d(v)=d(w)=2$, then $v, w \in N[B]$.

Proof. Assume to the contrary that some edge $v w$ has $v, w \notin N[B]$. By minimality, we can $L$-color $(G-v w)^{2}$. We uncolor $v$ and $w$. Since $v, w \notin N[B]$, both $v$ and $w$ have less than $\sqrt{k} \times \sqrt{k}$ colored neighbors in $G^{2}$. Since $|L(v)|=|L(w)|=k+2$, we can find distinct available colors for $v$ and $w$.

Suppose instead that $d(v)=d(w)=2$ and $v \in N[B]$ and $w \notin N[B]$. Again, by minimality we $L$-color $(G-v w)^{2}$, then uncolor $v$ and $w$. Now $v$ has at most $k+1$ colored neighbors in $G^{2}$, so $v$ has an available color. As before, we can color $w$. This gives an $L$-coloring for $G^{2}$, a contradiction.

Lemma 3.5. If $v w$ is an edge with $d(v)=d(w)=2$, then $v$ and $w$ have no common neighbor. 
Proof. Assume there exists a triangle $v w x$ with $d(v)=d(w)=2$. By minimality, we can $L$-color $(G \backslash\{v, w\})^{2}$. Both $v$ and $w$ have $d(x)-1 \leqslant k-1$ colored neighbors in $G^{2}$. So $v$ and $w$ each have at least 3 available colors, and thus we can color them both.

Lemma 3.6. Let $v x_{1} x_{2}$ be a triangle of $G$ such that some vertex $w \in S \backslash\left\{v, x_{1}, x_{2}\right\}$ has a common 2-neighbor with $x_{1}$. If either (a) $d\left(x_{2}\right)=2$ or (b) $d\left(x_{2}\right)=3$ and $w$ and $x_{2}$ have a common 2-neighbor, then $d\left(x_{1}\right) \geqslant 4$.

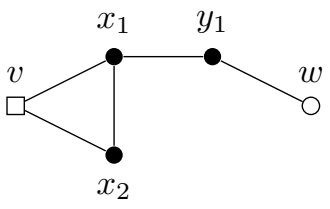

(a)

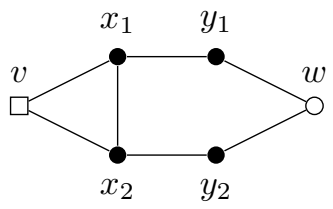

(b)

Figure 4: Forbidden configurations of Lemma 3.6

Proof. Let $y_{1}$ and $y_{2}$ denote the 2-neighbors of $w$ common with $x_{1}$ and $x_{2}$ if they exist (in Case (a), only $y_{1}$ is defined). Assume that $d\left(x_{1}\right)=3$. If $v w \in E(G)$, then $w v x_{1} y_{1}$ is a 4 -cycle in $G$, a contradiction. So $v w \notin E(G)$. By assumption, $w \notin\left\{v, x_{1}, x_{2}, y_{1}\right\}$. So if $w x_{2} \in E(G)$, then $w x_{2} x_{1} y_{1}$ is a 4-cycle in $G$, again a contradiction. Thus, $w x_{2} \notin E(G)$. Since $d\left(x_{1}\right)=3$ and $v, x_{2}, y_{1} \in N\left(x_{1}\right)$, we must have $w \notin N\left(x_{1}\right)$. Since $N\left(y_{1}\right)=\left\{x_{1}, w\right\}$, also $v y_{1} \notin E(G)$. So in both cases $w x_{1}, w x_{2}, w v, v y_{1} \notin E(G)$. And in (b) also $v y_{2} \notin E(G)$.

Let $S=\left\{x_{1}, x_{2}, y_{1}\right\}$ in Case (a), and $S=\left\{x_{1}, x_{2}, y_{1}, y_{2}\right\}$ in Case (b). By minimality, we $L$-color $(G \backslash S)^{2}$. For each $i \in\{1,2\}$, the number of colored neighbors in $G^{2}$ of $x_{i}$ is at most:

$$
|\{v, w\}|+\left|N(v) \backslash\left\{x_{1}, x_{2}\right\}\right| \leqslant 2+(k-2)=k .
$$

Thus, $x_{1}$ and $x_{2}$ both have at least 2 available colors, so we can color them. Further, for each $i \in\{1,2\}$, the number of colored neighbors of $y_{i}$ is at most

$$
\left|\left\{v, w, x_{1}, x_{2}\right\}\right|+\left|N(w) \backslash\left\{y_{i}\right\}\right| \leqslant 4+\sqrt{k}-1=\sqrt{k}+3 .
$$

Therefore, $y_{1}$ and $y_{2}$ (if defined) both have $k-\sqrt{k}-1$ available colors. Since $k$ is large enough, we can color them to get an $L$-coloring for $G^{2}$, a contradiction.

We combine Lemmas 3.4 and 3.6 to prove the reducibility of the bigger configuration shown in Figure 5 .

Lemma 3.7. Fix $v, w \in V(G)$ such that $w \in S$. Then the graph $G$ cannot contain distinct vertices $y_{1}, \ldots, y_{5}$ that are consecutive neighbors of $w$ and that satisfy both conditions below; see Figure 5 .

1. Each $y_{i}$ has degree two and has a common neighbor $x_{i}$ with $v$.

2. For each $i \in\{1, \ldots, 4\}$, each vertex inside cycle $v x_{i} y_{i} w y_{i+1} x_{i+1}$ is adjacent to $v$. 


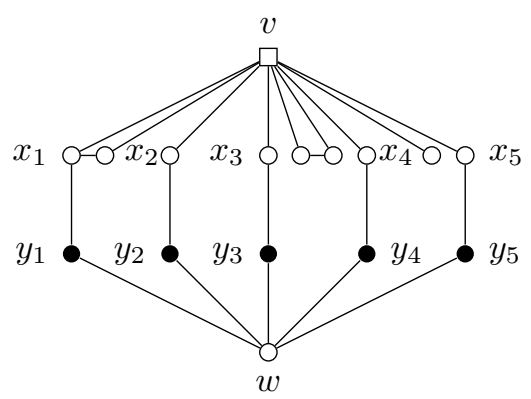

Figure 5: A possible configuration of Lemma 3.7.

Proof. We assume that $G$ contains such a configuration and reach a contradiction, by showing that $G$ contains a configuration forbidden by Lemma 3.6. Note that all $x_{i}$ 's are distinct, since $G$ contains no 4-cycle.

Below when we write a statement about $x_{i}$, we mean that it is true for each $i \in$ $\{2,3,4\}$. Since $w \in S$, Lemma 3.4 implies that $d\left(x_{i}\right) \geqslant 3$. Because $y_{1}, \ldots, y_{5}$ are consecutive neighbors of $w$, vertex $x_{i}$ is not adjacent to $w$. Since $G$ has no 4-cycle, $x_{i}$ has at most one common neighbor with $v$. Thus $d\left(x_{i}\right)=3$. Define $z$ so that $N\left(x_{3}\right)=\left\{v, y_{3}, z\right\}$. If $z \in\left\{x_{2}, x_{4}\right\}$, then $G$ contains the second configuration in Lemma 3.6, a contradiction. If $z$ has a neighbor other than $x_{3}$ and $v$, then call it $z^{\prime}$; now $z^{\prime}$ is adjacent to $v$ (by hypothesis 2), so $v x_{3} z z^{\prime}$ is a 4-cycle, a contradiction. Thus, $z$ is a 2 -vertex with $N(z)=\left\{x_{3}, v\right\}$. Now $G$ contains the first configuration in Lemma 3.6, again a contradiction.

\subsection{Outline of the proof}

Recall that $S$ is the set of small vertices, and $S_{i}$ is the set of small vertices with exactly $i$ big neighbors. Let $G^{\prime}$ denote the multigraph formed from $G$ by suppressing

$S, S_{i}$

$G^{\prime}$ every vertex of degree 2 in $S \backslash N[B]$, and then contracting every edge between $S_{1}$ and $B$. (Suppressing a 2-vertex $v$ means deleting $v$ and adding an edge between its two neighbors.) Note that $G^{\prime}$ may contain loops. For example, there is a loop in $G^{\prime}$ around a vertex $u$ if $u$ is a big vertex in $G$ and there is a triangle $u v w$ with $v, w \in S_{1}$. We say that a vertex of $G$ disappears when constructing $G^{\prime}$ if it is either a suppressed vertex, or a vertex in $S_{1}$.

Let $G^{\prime \prime}$ denote the multigraph formed from $G^{\prime}$ by removing every loop, and let $G^{\prime \prime \prime}$ denote the underlying multigraph of $G^{\prime \prime}$, i.e., the multigraph formed from $G^{\prime \prime}$ by deleting the minimal number of edges to remove all faces of length 2 . Note that $G^{\prime \prime \prime}$ can have parallel edges. For example, suppose $v$ and $w$ have parallel edges, say $e_{1}$ and $e_{2}$, in $G^{\prime}$. If some vertices are embedded inside and outside of the cycle $e_{1} e_{2}$, then in $G^{\prime \prime \prime}$ vertices $v$ and $w$ still have parallel edges, with those same vertices embedded inside and outside of the cycle $e_{1} e_{2}$. However, $G^{\prime \prime \prime}$ cannot have faces of length 2 .

An $r$-region of $G^{\prime \prime}$ is a set $\left\{f_{1}, \ldots, f_{r}\right\}$ of $r$ pairwise distinct faces of length 2 such that:

- For $1 \leqslant i<r, f_{i}$ shares one edge with $f_{i+1}$. (We say that the $f_{i}$ 's are consecutive.)
$G^{\prime \prime}$

$G^{\prime \prime \prime}$

$r$-region of $G^{\prime \prime}$

consecutive 
- All the $f_{i}$ 's have the same vertices $b_{1}, b_{2}$ on their boundary, where $b_{1}$ and $b_{2}$ are distinct vertices of $B$.

Note that each of the faces in an $r$-region is constructed from some cycle of $G$ when we apply the construction rules above. By extension, an $r$-region of $G$ is the subgraph of $G$ induced by the vertices of these cycles, together with those lying on the inside of those cycles. (We often simply write region, when the specific value of $r$ is less important.) When $R$ is an $r$-region of $G$, we say that $r$ is the size of $R$, and we denote by $V(R)$ the set of vertices appearing on all faces of $R$, excluding $b_{1}$ and $b_{2}$.

To reach a contradiction, we prove the following two propositions.

Proposition 3.8. $G$ contains an $r$-region with $r \geqslant \frac{\sqrt{k}}{50}-37$.

Proposition 3.9. $G$ does not contain any $r$-region for $r \geqslant 475353$.

Our contradiction now comes quickly. These propositions give that $\frac{\sqrt{k}}{50}-37<$ 475353. This inequality implies $k<23769500^{2}$, contradicting the hypothesis $k \geqslant \Delta_{0}=$ $23769500^{2}$.

We will devote a subsection to the proof of each proposition: Subsection 3.4 for Proposition 3.8 and Subsection 3.5 for Proposition 3.9. In Subsection 3.3, we prove structural lemmas about the regions in $G$.

\subsection{Structure of Regions}

We now classify each edge of $G^{\prime}$ based on its corresponding path (or cycle) in $G$. An edge $e$ in $G^{\prime}$ corresponds to a path or cycle $x_{1} \cdots x_{n}$ in $G$ if $e=x_{1} x_{n}$ and for each $i \in\{2, \ldots, n-1\}$, one of the following holds:

$r$-region of $G$

region

$V(R)$

- $x_{i}$ is a 2-vertex in $G$ and $x_{i-1}, x_{i+1} \in S$, or

- $x_{i} \in S_{1}$ and either $x_{i-1}$ or $x_{i+1}$ lies in $B$.

Due to the construction of $G^{\prime}$, for every loop (resp. non-loop edge) $e$ of $G^{\prime}$, there is a unique cycle (resp. path) $x_{1} \cdots x_{n}$ in $G$ corresponding to $e$ (with possibly $n=2$ ). Note that we used here that the suppressed 2-vertices are not in $N[B]$, hence every contracted edge (between $S_{1}$ and $B$ ) is between two adjacent vertices in $G$.

The following lemma ensures that every edge (resp. loop) of $G^{\prime}$ corresponds to a short path (resp. cycle) of $G$. It also gives a classification of all the possible such paths (resp. cycles), depicted in Figure6.
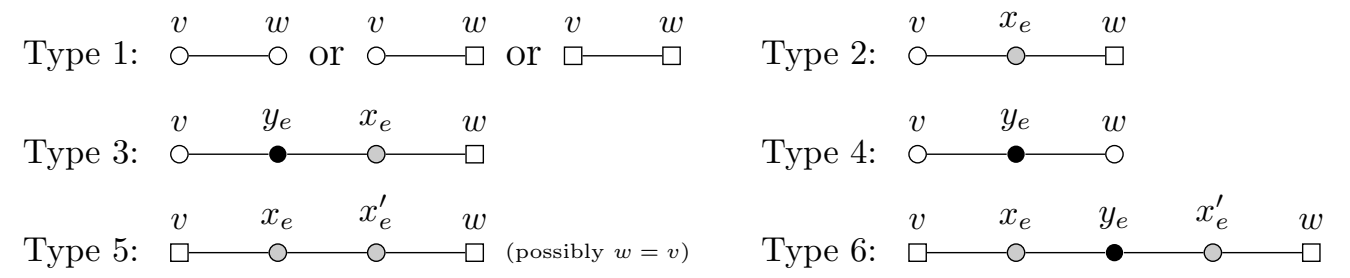

Figure 6: The six types of paths/cycles in $G$ that create edges in $G^{\prime}$ (gray vertices lie in $S_{1}$ ). 
Lemma 3.10. Each edge $e=v w$ of $G^{\prime}$ corresponds to a path or a cycle in $G$ for which exactly one of the following six conditions holds (up to exchanging $v$ with $w$ ). If e satisfies condition $i$ below (for some $i \in[6]$ ), then we say that e has type $i$. If $v \in S$, then e has one of types 1-4. If e is a loop of $G^{\prime}$, then e has type 5. Finally if $v, w \in B$, then e has type 1 , 5, or 6 .

1. $e \in E(G)$.

2. $w \in B$ and e corresponds to a path $v x_{e} w$ in $G$ with $x_{e} \in S_{1}$.

3. $w \in B$ and e corresponds to a path vy $y_{e} x_{e} w$ in $G$ with $x_{e} \in S_{1}$ and $d\left(y_{e}\right)=2$.

4. $w \in S$ and e corresponds to a path vyew in $G$ with $d\left(y_{e}\right)=2$.

5. e corresponds to a path or cycle $v x_{e} x_{e}^{\prime} w$ in $G$ with $x_{e}, x_{e}^{\prime} \in S_{1}$.

6. e corresponds to a path $v x_{e} y_{e} x_{e}^{\prime} w$ in $G$ with $x_{e}, x_{e}^{\prime} \in S_{1}$ and $d\left(y_{e}\right)=2$.

Proof. Due to the construction of $G^{\prime}$, each edge $e$ in $G^{\prime}$ between $v$ and $w$ comes from a path (or cycle) $P_{e}$ in $G$ between $v$ and $w$. In particular, every internal vertex of $P_{e}$ is either a 2-vertex in $S \backslash N[B]$ or a vertex of $S_{1}$ which is preceded or followed in $P_{e}$ by a big vertex. This implies that each internal vertex of $P_{e}$ is small, and that the only vertices of $P_{e}$ that can be big are $v$ and $w$.

By Lemma 3.4, no two consecutive vertices of $P_{e}$ are suppressed. This implies that $P_{e}$ has length at most four.

- If $P_{e}$ has length one, then $e$ has type 1.

- If $P_{e}$ has length two, then we have $v \neq w$ since $G$ is simple. Denote by $x$ the middle vertex of $P_{e}$. We must have either $v, w \in S$ and $d_{G}(x)=2$ (case 4 ), or $v \in B, w \in S$ and $x \in S_{1}$ (case 2 ).

- If $P_{e}$ has length three, then at least one of $v, w$ must be in $B$ and its neighbor in $P_{e}$ must be in $S_{1}$. If both $v$ and $w$ lie in $B$, then we are in case 5 ; otherwise, we have $v \neq w$ and we are in case 3 .

- Finally, if $P_{e}$ has length four, then we have $v \neq w$ since $G$ is $C_{4}$-free. Moreover, they both have to be big and their neighbors in $P_{e}\left(\right.$ say $\left.x_{e}, x_{e}^{\prime}\right)$ lie in $S_{1}$. The other vertex $y_{e}$ of $P_{e}$ must have degree two, so we are in case 6 .

Observe in particular that if $v$ is small, then cases 5 and 6 cannot occur. Moreover, if $v$ and $w$ are big, then only cases 1,5 , and 6 can occur. Finally, every loop of $G^{\prime}$ has type 5 .

In what follows, when referring to an edge $e$ with type $i$, we use $x_{e}, x_{e}^{\prime}$, and $y_{e}$ as defined in the corresponding part of Lemma 3.10. This lemma implies the following facts about the structure of regions in $G$.

Corollary 3.11. Let $R$ be a region of $G$. Then $V(R)$ is the disjoint union of three sets $B_{1}, B_{2}, D$ such that $B_{i} \subset N\left(b_{i}\right)$ for some $b_{1}, b_{2} \in B$, and $D$ is an independent set of 2-vertices, each with a neighbor in each of $B_{1}$ and $B_{2}$.

Proof. Let $R$ be a region of $G$. By definition, there exists $b_{1}, b_{2} \in B$ on the boundary of every face of $R$ in $G^{\prime \prime}$. Therefore, in $G^{\prime}$, the edges appearing in $R$ are either loops on $b_{1}$ or $b_{2}$ or edges between $b_{1}$ and $b_{2}$. 
Note that $V(R)$ is the set of all vertices of $G$ that disappear when we construct the edges of $R$ in $G^{\prime}$. For each $i \in\{1,2\}$, define $B_{i}$ as the set of vertices $v$ of $G$ such that $v b_{i}$ is contracted when constructing an edge of $R$ in $G^{\prime}$. We also define $D$ as the set of vertices in $G$ that are suppressed when constructing an edge of $R$ in $G^{\prime}$. By definition, we have $B_{i} \subset N\left(b_{i}\right)$

By Lemma 3.10, since $b_{1}, b_{2} \in B$, each edge $e$ between $b_{1}$ and $b_{2}$ in $G^{\prime}$ has type 1 , 5 , or 6 , and each loop around $b_{1}, b_{2}$ has type 5. This ensures that $V(R)=B_{1} \cup B_{2} \cup D$ and that $D$ contains only vertices of degree 2 in $G$. Using again Lemma 3.10 , this implies that $D$ is an independent set.

It remains to show that these sets are pairwise disjoint. Assume that there is $x \in B_{1} \cap B_{2}$. Now $x b_{1}$ and $x b_{2}$ are both contracted when constructing $G^{\prime}$. This requires that $x \in S_{1}$. Since $b_{1}$ and $b_{2}$ are both big, we must have $b_{1}=b_{2}$, a contradiction. Further, since $b_{1} \in B$, no neighbor of $b_{1}$ is suppressed during the construction of $G^{\prime}$. Since $B_{1} \subset N\left(b_{1}\right)$, we thus have $D \cap B_{1}=\varnothing$. By symmetry, we also have $D \cap B_{2}=\varnothing$.

In the following, given a region $R$, we use the notation of Corollary 3.11.

\subsection{Proof of Proposition 3.8: $G$ has Large Regions}

Our goal in this subsection is to find a large region in $G$. To this end, we look for a large set of consecutive faces of length 2 in $G^{\prime}$. We first recall a result from [3] (Lemma 3.6 in that paper) allowing us to find a vertex in $G^{\prime}$ with few neighbors in $G^{\prime \prime \prime}$.

Lemma $3.12([3])$. There exists $b_{1} \in B$ such that $d_{G^{\prime \prime \prime}}\left(b_{1}\right) \leqslant 40$ and $d_{G^{\prime \prime \prime}[B]}\left(b_{1}\right) \leqslant 10$.

We note that the general context of [3] is planar graphs with girth at least 5 . However, the proof of Lemma 3.12 uses only that $G$ has no 4-cycles.

Our goal is to apply a pigeonhole-like argument to find a large number of consecutive edges between two vertices in $G^{\prime \prime}$. To this end, we first need to control the degrees of vertices in $G^{\prime \prime}$. We begin with a definition. The half-edges of $G^{\prime}$ are the elements of the multiset of pairs $(u, e)$ where $e$ is an edge incident to $u$. Note that when $e$ is a loop around $u$, there are still two half-edges $(u, e)$. Observe also that since we fixed a plane embedding of $G$, there is a natural cyclic ordering of the half-edges around each fixed vertex $u$.

Lemma 3.13. If e is a loop around a vertex $v$ in $G^{\prime}$, then one of the half-loops induced by e must be followed or preceded by a half-edge $(v, v w)$ with $v \neq w$.

Proof. By Lemma 3.10, every loop has type 5. So let $x_{e}$ and $x_{e}^{\prime}$ denote the vertices in $G$ that merged into $v$ to form $e$ in $G^{\prime}$. By Lemma 3.5, either $d\left(x_{e}\right)>2$ or $d\left(x_{e}^{\prime}\right)>2$; by symmetry, assume $d\left(x_{e}\right)>2$. Among all neighbors of $x_{e}$ in $G$, other than $x_{e}^{\prime}$ and $v$, choose $w$ to be one that immediately precedes or follows $x_{e}^{\prime}$.

If $w$ is not suppressed in $G^{\prime}$, then the half-edge $(v, v w)$ precedes or follows $(v, e)$ or $\left(v, e^{\prime}\right)$. Note that $v w \notin E(G)$ since otherwise $v w x_{e} x_{e}^{\prime}$ is a 4 -cycle in $G$. Thus we have $v \neq w$ in $G^{\prime}$ and the lemma is true. So assume that $w$ is suppressed. Now $w$ has degree 2 in $G$. Let $x$ be the neighbor of $w$ other than $x_{e}$. Since $x_{e}$ is small, Lemma 3.4 ensures 
that $x$ has degree at least 3 in $G$; hence, it is not suppressed in $G^{\prime}$. Therefore, the half-edge $(v, v x)$ precedes or follows $(v, e)$ or $\left(v, e^{\prime}\right)$. Again, $v x \notin E(G)$ since otherwise $v x w x_{e}$ is a 4 -cycle in $G$. Thus $x \neq v$ in $G^{\prime}$ and the lemma is true.

Lemma 3.13 implies the following relationship between degrees of vertices in $G^{\prime \prime}$ and in $G^{\prime}$.

Corollary 3.14. Every $v \in V\left(G^{\prime}\right)$ satisfies $d_{G^{\prime \prime}}(v) \geqslant \frac{d_{G^{\prime}}(v)}{5}$.

Proof. Suppose $v \in V\left(G^{\prime}\right)$ and consider the half-edges around $v$ in $G^{\prime}$. By definition, there are $d_{G^{\prime}}(v)$ half-edges around $v$ and $d_{G^{\prime \prime}}(v)$ of them are not half-loops. So it suffices to prove that the number of half-loops around $v$ is at most four times the number of the other half-edges, i.e., at most $4 d_{G^{\prime \prime}}(v)$.

Suppose $w \in N_{G^{\prime}}(v)$. Consider the two half-edges $(v, e)$ and $(v, f)$ such that $(v, e)$, $(v, v w)$ and $(v, f)$ are consecutive around $v$. Let $F(w)$ be the maximum subset of $\{(v, e),(v, f)\}$ containing only half-loops. Lemma 3.13 ensures that, for every loop, one of its half-loops appears in $F(w)$ for some $w \in N_{G^{\prime}}(v)$. Therefore, the number of half-loops around $v$ is at most

$$
2\left|\cup_{w \in N_{G^{\prime}}(v)} F(w)\right| \leqslant 4\left|N_{G^{\prime}}(v)\right|=4 d_{G^{\prime \prime}}(v) .
$$

This concludes the proof, since

$$
d_{G^{\prime}}(v) \leqslant d_{G^{\prime \prime}}(v)+4 d_{G^{\prime \prime}}(v)=5 d_{G^{\prime \prime}}(v) .
$$

Consider the vertex $b_{1}$ obtained by Lemma 3.12, By Corollary 3.14, we have

$$
d_{G^{\prime \prime}}\left(b_{1}\right) \geqslant \frac{d_{G^{\prime}}\left(b_{1}\right)}{5} \geqslant \frac{d_{G}\left(b_{1}\right)}{5} \geqslant \frac{\sqrt{k}}{5}
$$

Using a pigeonhole argument, we will see that $b_{1}$ has some neighbor $b_{2}$ such that at least $\frac{\sqrt{k}}{5 \times 40}$ consecutive edges incident to $b_{1}$ end at $b_{2}$. Note that Proposition 3.8 almost follows from this result (with $\frac{\sqrt{k}}{50}$ replaced by $\frac{\sqrt{k}}{200}$ ). We only need to refine this argument to show how to force $b_{2} \in B$, i.e., $b_{2} \notin S^{\prime}$, where $S^{\prime}=V\left(G^{\prime}\right) \backslash B$. To this end, we show that small vertices are incident to few consecutive edges in $G^{\prime \prime}$.

Lemma 3.15. If $v \in B$ and $w \in S^{\prime}$, then $(v, w)$ is on the boundary of at most 8 consecutive faces of length 2 in $G^{\prime \prime}$.

Proof. Pick $v \in B$ such that there is an edge $v w \in E\left(G^{\prime}\right)$, with $w \in S^{\prime}$. We consider each possible type of edge in $G^{\prime}$ between $v$ and $w$. The type 3 edges are a special case, which we postpone to the end. Since $G$ is simple, at most one edge $v w$ of $G^{\prime}$ has type 1. Similarly, if $G^{\prime}$ has two edges $e_{1}$ and $e_{2}$ of type 2 , then $x_{e_{1}} \neq x_{e_{2}}$. Thus $v x_{e_{1}} w x_{e_{2}}$ is a 4 -cycle in $G$, a contradiction. So $G^{\prime}$ has at most one edge of type 2 . Since $v \in B$ and $w \in S^{\prime}, G^{\prime}$ has no edge of type 4,5 , or 6 .

Only type 3 edges remain. We assume such an edge exists, since otherwise the lemma holds. Note that $G^{\prime}$ has no edge of type 4 (since $v \in B$ ), nor of type 1 (since $G$ has no 4 -cycle), nor of type 5 or 6 (since $w \in S^{\prime}$ ). So $G^{\prime}$ has at most one edge $f$ not of type 3 , and $f$, if it exists, has type 2 . Thus, edge $f$ separates two blocks of consecutive 
type 3 edges. To prove the lemma, it suffices to prove that each such block has size at most four.

Assume that $e_{1}, \ldots, e_{5}$ are edges of type 3 that are consecutive in $G^{\prime \prime}$. We now prove that the hypotheses of Lemma 3.7 are satisfied by the subgraph of $G$ induced by the vertices inside the cycle $v x_{e_{1}} y_{e_{1}} w y_{e_{5}} x_{e_{5}}$. Since each edge $e_{i}$ has type 3 , the first hypothesis holds.

To prove the second hypothesis holds, assume that some vertex $x$ is not adjacent to $v$, but $x$ lies inside some cycle $C=v x_{e_{i}} y_{e_{i}} w y_{e_{i+1}} x_{e_{i+1}}$. Note that $x$ is not a neighbor of $y_{e_{i}}$ or $y_{e_{i+1}}$, since they both have degree 2 ; nor of $w$ since $e_{i}$ and $e_{i+1}$ are consecutive edges in $G^{\prime \prime}$. Note that $e_{i}$ and $e_{i+1}$ bound a face of length 2 in $G^{\prime \prime}$ so every vertex inside the cycle $C$ disappears when we construct $G^{\prime}$. Thus, all these vertices are small, and either lie in $S_{1}$ or lie in $S \backslash N[B]$ and have degree 2 in $G$. Hence, $v$ is the only big vertex inside or on $C$ and $x v \notin E(G)$; so $x \notin \cup_{i \geqslant 1} S_{i}$.

Since $x \notin S_{1}, x$ has degree 2 and its two neighbors, say $y$ and $z$, lie in $S$. Applying Lemma 3.4 to edges $x y$ and $x z$, we get that $y, z \in N[B]$. This implies that both $y$ and $z$ are neighbors of $v$, so $x y v z$ is a 4 -cycle in $G$, a contradiction. Therefore, no such $x$ exists.

Now Lemma 3.7 yields a contradiction, since $G$ cannot contain this configuration.

We can now finish the proof of Proposition 3.8 .

Proof of Proposition 3.8. Let $b_{1}$ be a vertex in $G^{\prime \prime \prime}$ guaranteed by Lemma 3.12. For each small neighbor $v$ of $b_{1}$ in $G^{\prime \prime \prime}$ and edge $v b_{1}$, Lemma 3.15 ensures that in $G^{\prime \prime}$ edge $v b_{1}$ corresponds to at most 9 edges between $b_{1}$ and $v$. Since $d_{G^{\prime \prime \prime}}\left(b_{1}\right) \leqslant 40$, the number of such edges is at most $9 \times 40=360$. However, by Corollary 3.14, we have $d_{G^{\prime \prime}}\left(b_{1}\right) \geqslant \frac{d_{G}\left(b_{1}\right)}{5} \geqslant \frac{\sqrt{k}}{5}$. Thus, there must exist a big neighbor $b_{2}$ of $b_{1}$ in $G^{\prime \prime}$ such that there are at least $\frac{\frac{\sqrt{k}}{5}-360}{d_{G^{\prime \prime \prime}[B]}\left(b_{1}\right)} \geqslant \frac{\sqrt{k}}{50}-36$ consecutive edges $b_{1} b_{2}$ in $G^{\prime \prime}$. By definition, these edges form a region of size $\frac{\sqrt{k}}{50}-37$ in $G$.

\subsection{Proof of Proposition 3.9: Large Regions are Reducible}

In this section, we show that $G$ cannot contain arbitrarily large regions, i.e., for $r$ large enough every $r$-region is reducible. Note that the square of such $r$-regions consists of two cliques, with some edges between them. Following the terminology of Corollary 3.11. we denote the vertices of these cliques by $B_{1}$ and $B_{2}$. As before, $D$ denotes a set of independent 2-vertices, each with one neighbor in $B_{1}$ and one neighbor in $B_{2}$. We begin by proving that there are only few edges between $B_{1}$ and $B_{2}$.

Lemma 3.16. Let $R$ be an $r$-region of $G$. Each $w \in B_{1} \cup B_{2}$ has at most one neighbor in $B_{1}$, at most one in $B_{2}$, and at most eight in $D$.

Proof. Suppose $w \in B_{1} \cup B_{2}$. If $w$ has two neighbors $x$ and $y$ in $B_{i}$, then $b_{i} x w y$ is a 4-cycle in $G$, a contradiction. So we assume $w$ has at most one neighbor in each of $B_{1}$ and $B_{2}$. In what follows, we assume by symmetry that $w \in B_{1}$. 
Suppose that $w$ has 5 consecutive neighbors $x_{1}, \ldots, x_{5}$, all in $D$, and denote by $y_{i}$ the common neighbor of $x_{i}$ and $b_{2}$. By Lemma 3.7, there is a vertex $z$ inside some cycle $w x_{i} y_{i} b_{2} y_{i+1} x_{i+1}$ that is not adjacent to $b_{2}$. Since $R$ is an $r$-region, $z$ disappears when we construct $G^{\prime}$. Since $z \notin N_{G}\left(b_{2}\right)$, vertex $z$ must be a 2-vertex. By Lemma 3.4 . each neighbor of $z$ is adjacent to $b_{2}$. So $G$ contains a 4-cycle, a contradiction. Thus, $w$ has at most 4 consecutive neighbors in $D$.

Consider an edge $w x$ between these blocks of consecutive neighbors in $D$ where $x \in V(R) \backslash D$. Then $x$ cannot lie in $B_{1}$, otherwise $b_{1} w x$ is a triangle not containing $b_{2}$ nor any vertex in $B_{2}$. By planarity, there cannot be vertices of $D$ inside and outside of this triangle. Therefore $x \in B_{2}$.

Since $G$ has no 4-cycle, at most one such neighbor $x$ exists, so $w$ has at most two blocks of consecutive neighbors in $D$. This proves the final assertion.

We note that we can prove Theorem 3.1 more simply (and with a better bound on $\Delta$ ) if we only want the result for list-coloring. In fact, we do this in Section 3.6. where we prove it for correspondence coloring. However, to prove this same bound for Alon-Tarsi number, as we do below, seems to require using the Kernel Lemma (Lemma 3.17).

Proving that $G$ does not contain large regions amounts to proving that $r$-regions of $G$ are square $L^{\prime}$-colorable for a suitable assignment $L^{\prime}$. To prove this new assertion, we use an auxiliary result about choosability, due to Bondy, Boppana, and Siegel (see Remark 2.4 in [1]). This result applies to kernel perfect digraphs. We briefly recall the definition here. A kernel $K$ in a digraph $D$ is a subset of $V(D)$ such that every vertex $v$ of $D$ satisfies: $v \in K$ if and only if $N^{+}(v) \cap K=\varnothing$. A digraph is kernel perfect if each of its induced subgraphs has a kernel.

Lemma 3.17. Let $D$ be a kernel perfect digraph $D$ with underlying graph $H$. If $L$ is a list assignment for $V(H)$ such that for all $v \in V(H),|L(v)| \geqslant d^{+}(v)+1$, then $H$ is L-colorable.

We use this lemma to reduce the problem of square $L$-coloring an $r$-region to finding a kernel perfect orientation. We apply this method to prove the following generic result about choosability of graphs covered by two cliques with few edges between them. (Our next lemma is analogous to Lemma 3.13 in [3]. One major reason that our bounds below on $\left|B_{i}\right|$ and $\left|T_{i}\right|$ are so much larger is that here we apply the lemma to a graph $G$ that can contain triangles.)

Lemma 3.18. Let $H$ be a graph covered by two disjoint cliques, $B_{1}$ and $B_{2}$. Let $L$ be a list assignment for $V(H)$ and suppose $T_{i} \subset B_{i}$ for each $i \in\{1,2\}$. Now $H$ is $L$-colorable if the following five conditions hold.

1. $\left|B_{1}\right| \geqslant 52811$ and $\left|B_{2}\right| \geqslant 52811$.

2. $\left|T_{1}\right| \leqslant 4400$ and $\left|T_{2}\right| \leqslant 4400$.

3. For each $v \in B_{i},\left|N(v) \cap B_{3-i}\right| \leqslant 11$.

4. For each $v \in T_{i},|L(v)| \geqslant\left|B_{i}\right|-44$.

5. For each $v \in B_{i} \backslash T_{i},|L(v)| \geqslant\left|B_{i}\right|$. 
Proof. To prove this result we construct an orientation $D$ of $H$ such that $D$ satisfies the hypotheses of Lemma 3.17 . We first show that we can order the vertices $x_{1}, \ldots, x_{\left|B_{1}\right|}$ and $y_{1}, \ldots, y_{\left|B_{2}\right|}$ of $B_{1}$ and $B_{2}$ such that $T_{1}=\left\{x_{1}, \ldots, x_{\left|T_{1}\right|}\right\}, T_{2}=\left\{y_{1}, \ldots, y_{\left|T_{2}\right|}\right\}$ and every path beginning and ending in $\left\{x_{\left|B_{1}\right|-10}, \ldots, x_{\left|B_{1}\right|}, y_{\left|B_{2}\right|-10}, \ldots, y_{\left|B_{2}\right|}\right\}$ that alternates between $B_{1}$ and $B_{2}$ has length at least 4 . Note that a single edge may be an alternating path, so we require that no edge joins $x_{i}$ and $y_{j}$ whenever $i \geqslant\left|B_{1}\right|-10$ and $j \geqslant\left|B_{2}\right|-10$.

\section{Definition of the Orderings}

We now construct the vertex orderings in the previous paragraph. Their only nontrivial property is the absence of short alternating paths between the final 11 vertices in $B_{1}$ and those in $B_{2}$. So, our goal is to construct $Z_{1} \subset B_{1}$ and $Z_{2} \subset B_{2}$ with $\left|Z_{1}\right|=\left|Z_{2}\right|=11$ such that no alternating path of length at most 3 begins in $Z_{1}$ and ends in $Z_{2}$. To this end, we first define $Z_{2}$, then count the number of vertices in $B_{1}$ reachable from $Z_{2}$ with such an alternating path.

If there exists $v \in B_{1} \backslash N\left(T_{2}\right)$ with 11 neighbors in $B_{2}$, then we take $Z_{2}=N_{H}(v) \cap B_{2}$. If no such vertex exists, then we swap the roles of $B_{1}$ and $B_{2}$, take $Z_{2}$ as any subset of $B_{2} \backslash\left(T_{2} \cup N\left(T_{1}\right)\right)$ of size 11 (this is always possible since $\left|B_{2}\right| \geqslant 52811 \geqslant\left|T_{2}\right|+$ $\left.11\left|T_{1}\right|+11\right)$, and let $v$ be any vertex of $B_{1}$. Since every element of $Z_{2}$ has at most 10 neighbors in $B_{1} \backslash\{v\}$, we have $\left|N_{B_{1}}\left(Z_{2}\right) \backslash\{v\}\right| \leqslant 11 \times 10=110$. Moreover, each vertex in $N_{B_{1}}\left(Z_{2}\right) \backslash\{v\}$ has at most 11 neighbors in $B_{1}$ (one of them being in $Z_{2}$ ). Since the only neighbors of $v$ in $B_{2}$ are in $Z_{2}$, we obtain

$$
\left|N_{B_{2}}\left(N_{B_{1}}\left(Z_{2}\right)\right) \backslash Z_{2}\right| \leqslant 11 \times 10^{2}=1100 .
$$

By the same argument, the set of vertices of $B_{1}$ reachable from $Z_{2}$ with an alternating path of length exactly 3 has size

$$
\left|N_{B_{1}}\left(N_{B_{2}}\left(N_{B_{1}}\left(Z_{2}\right)\right) \backslash Z_{2}\right)\right| \leqslant 1100 \times 10=11000 .
$$

So the number of vertices of $B_{1}$ that are excluded from appearing in $Z_{1}$, because of paths to $Z_{2}$, is at most

$$
\left|N_{B_{1}}\left(N_{B_{2}}\left(N_{B_{1}}\left(Z_{2}\right)\right) \backslash Z\right)\right|+\left|N_{B_{1}}\left(Z_{2}\right) \backslash\{v\}\right|+|\{v\}|=11000+110+1=11111 .
$$

Further, we must also remove vertices of $T_{1}$. Thus, we can choose $Z_{1}$ as desired, since $\left|B_{1}\right|-\left|T_{1}\right|-11111 \geqslant 11$.

\section{Definition of the Orientation}

For each edge with both endpoints in the same clique, direct it toward the vertex of lower index. For every other edge, direct it in both directions, unless one of its endpoints is among the last 11 vertices of $B_{1}$ or $B_{2}$. In this case, direct the edge toward this endpoint. 


\section{The Orientation is Kernel-perfect}

Let $A \subseteq V(H)$, with $A \neq \varnothing$. We look for a kernel of $A$. Let $x_{p}$ (resp. $y_{q}$ ) denote the vertex with smallest index in $A \cap B_{1}$ (resp. $A \cap B_{2}$ ), if it exists. If $A \cap B_{1}=\varnothing$, then $\left\{y_{q}\right\}$ is a kernel. Similarly, if $A \cap B_{2}=\varnothing$, then $\left\{x_{p}\right\}$ is a kernel. So we assume that both $x_{p}$ and $y_{q}$ are well-defined. We can also assume that $x_{p} y_{q} \in E(H)$, since otherwise $\left\{x_{p}, y_{q}\right\}$ is a kernel.

Let $x_{r}$ (resp. $\left.y_{s}\right)$ denote the vertex with smallest index in $A \cap B_{1}$ (resp. $A \cap B_{2}$ ) that is not a neighbor of $y_{q}$ (resp. $x_{p}$ ).

We now prove that at least one of $\left\{x_{p}\right\},\left\{x_{p}, y_{s}\right\},\left\{y_{q}\right\}$ and $\left\{x_{r}, y_{q}\right\}$ is a kernel. Assume the contrary. Since $\left\{x_{p}, y_{s}\right\}$ is not a kernel, there exists $y_{j}$ such that $q \leqslant j<s$ and either there is no edge $x_{p} y_{j}$ or it is directed only towards $y_{j}$. Due to the choice of $s$, this edge is present in $H$ and is thus directed only one way. (If $y_{s}$ is not well-defined, i.e. if $x_{p}$ is adjacent to every vertex in $A \cap B_{2}$, we can obtain the same result using that $\left\{x_{p}\right\}$ is not a kernel.)

Similarly, using that $\left\{x_{r}, y_{q}\right\}$ is not a kernel (or only $\left\{y_{q}\right\}$ if $y_{q}$ is adjacent to every vertex in $A \cap B_{1}$ ), we have an edge $x_{i} y_{q}$ directed only towards $x_{i}$.

Since $x_{i} y_{q}$ and $x_{p} y_{j}$ are directed towards $x_{i}$ and $y_{j}$, this ensures that $x_{i}$ and $y_{j}$ are both among the final 11 vertices of $B_{1}$ and $B_{2}$. However, this is impossible, since $x_{i} y_{q} x_{p} y_{j}$ would be a path of length 3 that alternates between $B_{1}$ and $B_{2}$ and begin and ends in the final 11 vertices of $B_{1}$ and $B_{2}$. Thus, either $\left\{x_{p}, y_{s}\right\},\left\{x_{p}\right\},\left\{x_{r}, y_{q}\right\}$ or $\left\{y_{q}\right\}$ is a kernel of $A$. So the orientation is kernel-perfect.

\section{The Orientation has Small Out-degrees}

We now prove that $|L(v)| \geqslant d^{+}(v)+1$ for every $v \in V(H)$. By symmetry, it suffices to prove this for all $v \in B_{1}$, i.e., $v=x_{i}$ whenever $i \in\left\{1, \ldots,\left|B_{1}\right|\right\}$. If $i \leqslant\left|T_{1}\right|$, i.e., $v \in T_{1}$, then $v$ has at most $\left|T_{1}\right|-1 \leqslant 4399$ out-neighbors in $B_{1}$ and at most 11 out-neighbors in $B_{2}$. So $d^{+}(v)+1 \leqslant 4411 \leqslant\left|B_{1}\right|-44 \leqslant|L(v)|$. If $\left|T_{1}\right|<i \leqslant\left|B_{1}\right|-11$, then $v$ has at most $\left|B_{1}\right|-12$ out-neighbors in $B_{1}$ and at most 11 in $B_{2}$. So $d^{+}(v)+1 \leqslant\left|B_{1}\right| \leqslant|L(v)|$. If $i>\left|B_{1}\right|-11$, then every out-neighbor of $v$ is in $B_{1}$, so $d^{+}(v)+1 \leqslant\left|B_{1}\right| \leqslant|L(v)|$.

We now use this lemma to prove Proposition 3.9, i.e., that large regions are reducible for square choosability.

Proof of Proposition 3.9. We use proof by contradiction. Assume that $G$ has an $r$ region $R$ with $r \geqslant 475353$. Let $v_{1}$ and $v_{2}$ be adjacent vertices of $R$ such that any vertex at distance 2 in $G$ from $\left\{v_{1}, v_{2}\right\}$ lies in $\left\{b_{1}, b_{2}\right\} \cup V(R) \cup N\left(b_{1}\right) \cup N\left(b_{2}\right)$. To see that such vertices exist, pick $v_{1} \in B_{1}$ such that each face containing $v_{1}$ is in $R$, and let $v_{2}$ be a neighbor of $v_{1}$ in $B_{2} \cup D$.

Let $T$ denote the set of vertices in $B_{1} \cup B_{2}$ that appear on a face of $G$ not in $R$. Note that $|T| \leqslant 4$; this is because each vertex of $T$ must lie on the first or last edge of the $r$-region in $G^{\prime}$, and each of these edges has exactly one vertex in each of $B_{1}$ and $B_{2}$. Let $T^{(1)}=N(T) \cap V(R), T^{(2)}=N\left(T^{(1)}\right) \cap V(R)$ and $T^{(3)}=N\left(T^{(2)}\right) \cap V(R)$, so that for $1 \leqslant i \leqslant 3, T \cup \cdots \cup T^{(i)}$ is the set of vertices of $V(R)$ at distance at most $i$ from $T$ (with the distance taken in $V(R)$ ). By Lemma 3.16, each vertex of $T$ has at most 10 neighbors in $V(R)$, so $\left|T^{(1)}\right| \leqslant 40,\left|T^{(2)}\right| \leqslant 400$ and $\left|T^{(3)}\right| \leqslant 4000$. 
By minimality, $\left(G-v_{1} v_{2}\right)^{2}$ has an $L$-coloring $\varphi$. Let $B_{i}^{\prime}=B_{i} \backslash N[T]$. We uncolor $\varphi, B_{i}^{\prime}$ the vertices of $B_{1}^{\prime} \cup B_{2}^{\prime} \cup D$. We also define $T_{i}$ as the set of vertices of $B_{i}^{\prime}$ with some colored neighbor from $V(R)$ in $G^{2}$, i.e., $T_{i}=B_{i}^{\prime} \cap\left(T^{(2)} \cup T^{(3)}\right)$. Finally, let $H=G^{2}\left[B_{1}^{\prime} \cup B_{2}^{\prime}\right]$. Note that $B_{1}^{\prime}$ and $B_{2}^{\prime}$ are cliques in $H$. Moreover, they are disjoint since $B_{1}^{\prime} \cap B_{2}^{\prime} \subset B_{1} \cap B_{2}=\varnothing$.

Our goal is now to apply Lemma 3.18 to $L^{\prime}$-color $H$, where $L^{\prime}$ is the list assignment formed from $L$ by removing all colors already used on vertices at distance at most 2 :

$$
L^{\prime}(v)=L(v) \backslash\left\{\varphi(w), w \in N^{2}(v) \backslash(V(H) \cup D)\right\} .
$$

We prove that the hypotheses of Lemma 3.18 are satisfied.

Suppose $v \in B_{1}^{\prime}$. Now $\left|N^{2}(v) \cap B_{2}^{\prime}\right|=\left|N(v) \cap B_{2}^{\prime}\right|+\sum_{w \in N(v)}\left|N(w) \cap B_{2}^{\prime}\right|$. By Lemma 3.16, for each $w \in V(R),\left|N(w) \cap B_{2}^{\prime}\right| \leqslant 1$. Moreover, if $w \notin V(R)$, then $\left|N(w) \cap B_{2}^{\prime}\right|=0$, unless $w=b_{2}$. Since $b_{2} \notin N(v)$, we get

$$
\left|N^{2}(v) \cap B_{2}^{\prime}\right| \leqslant 1+|N(v) \cap V(R)| \leqslant 11 .
$$

Suppose $v \in B_{1}^{\prime} \backslash T_{1}$. By definition, $v$ is distance at least four from $T$ (in $V(R)$ ), hence at distance at least three (in $V(R)$ ) from $N[T]$, the set of colored vertices of $V(R)$. So the only colored neighbors of $v$ in $G^{2}$ are in $\left\{b_{1}, b_{2}\right\} \cup\left(N\left(b_{1}\right) \backslash B_{1}^{\prime}\right)$. Hence, we have

$$
\left|L^{\prime}(v)\right| \geqslant k+2-\left(2+k-\left|B_{1}^{\prime}\right|\right)=\left|B_{1}^{\prime}\right| .
$$

Suppose $v \in T_{1}$. By construction, its colored neighbors in $G^{2}$ are in $\left\{b_{1}, b_{2}\right\} \cup$ $\left(N\left(b_{1}\right) \backslash B_{1}^{\prime}\right) \cup T \cup T^{(1)}$. Since $|T|+\left|T^{(1)}\right| \leqslant 44$, we have $\left|L^{\prime}(v)\right| \geqslant\left|B_{1}^{\prime}\right|-44$.

We already saw that $\left|T_{1}\right| \leqslant\left|T^{(2)} \cup T^{(3)}\right| \leqslant 400+4000=4400$. There are $r+1$ edges in the region $R$ (in $G^{\prime}$ ). Every such edge (except $b_{1} b_{2}$ if it exists) corresponds to a path containing a vertex in $B_{1}$. By Lemma 3.16, each vertex in $B_{1}$ accounts for at most nine of them. Therefore, $\left|B_{1}\right| \geqslant \frac{r}{9}$. Observe also that $\left|N[T] \cap B_{1}\right| \leqslant 6$ since $\left|T \cap B_{1}\right|=2$ and, by Lemma 3.16 , every vertex of $B_{1} \cup B_{2}$ has at most one neighbor in each of $B_{1}$ and $B_{2}$. We thus obtain:

$$
\left|B_{1}^{\prime}\right| \geqslant\left|B_{1}\right|-\left|N[T] \cap B_{1}\right| \geqslant \frac{r}{9}-6 \geqslant 52811 .
$$

We can thus apply Lemma 3.18 to find an $L^{\prime}$-coloring of $H$.

It remains to color the vertices in $D$. Note that each has at most $2 \sqrt{k}$ neighbors and $k+2$ colors. So we can greedily color the vertices in $D$.

This completes the proof of Theorem 3.1 .

\subsection{Extension to correspondence coloring}

In this section, we prove the following extension of Theorem 3.1 to correspondence coloring. (Recall the definition of correspondence coloring from the end of Section 2.1.)

Theorem 3.19. There exists $\Delta_{0}$ such that if $G$ is a plane graph with no 4-cycles and with $\Delta(G) \geqslant \Delta_{0}$, then $\chi_{\text {corr }}\left(G^{2}\right) \leqslant \Delta+2$. 
Let $\Delta_{0}=2642900^{2}=6984920410000$, and fix $k \geqslant \Delta_{0}$. We prove Theorem 3.19

by contradiction. Suppose the theorem is false; let $G$ be a counterexample minimizing $|V(G)|+|E(G)|$, and let $C$ be a $(k+2)$-correspondence assignment for $G^{2}$ such that $G^{2}$ has no $C$-coloring. So $C$ assigns, to each pair of vertices $(v, w)$ adjacent in $G^{2}$, a partial matching $C_{v w}$ between $\{v\} \times\{1, \ldots, k+2\}$ and $\{w\} \times\{1, \ldots, k+2\}$.

We claim that Lemmas 3.2 through 3.16 still hold for $G$ in this new setting, since in proving each lemma we color vertices using only that they have more available colors than colored neighbors. So Proposition 3.8 also still holds. It thus suffices to prove the following generalization of Proposition 3.9 for $G$.

Proposition 3.20. Every $r$-region of $G$ satisfies $r \leqslant 52821$.

Assuming this proposition holds, we can conclude. Indeed, Propositions 3.8 and 3.20 imply that $\frac{\sqrt{k}}{50}-37<52821$, i.e., that $k<2642900^{2}=6984920410000=\Delta_{0}$, a contradiction.

It thus remains to prove that large regions are reducible, by generalizing Lemma 3.18 The argument using kernel-perfect orientations is no longer valid, since Lemma 3.17 does not extend to correspondence coloring.

Lemma 3.21. Let $H$ be a graph covered by two disjoint cliques, $B_{1}$ and $B_{2}$, each of size $n$. Suppose there exist $T_{1} \subset B_{1}$ and $T_{2} \subset B_{2}$, and a function $f$ satisfying the four properties below. If $n \geqslant 5863$, then every $f$-correspondence assignment $C$ admits a C-coloring.

1. For each $v \in\left(B_{1} \backslash T_{1}\right) \cup\left(B_{2} \backslash T_{2}\right)$, we have $f(v) \geqslant n$.

2. For each $v \in T_{1} \cup T_{2}$, we have $f(v) \geqslant n-44$.

3. $\left|T_{1}\right| \leqslant 4400$ and $\left|T_{2}\right| \leqslant 4400$.

4. $\Delta(H)-n+1 \leqslant 11$.

Proof. Let $A$ be a subset of $B_{1} \backslash T_{1}$ with $|A|=\Delta(H)+1-n$. Since each vertex $v \in\left(B_{1} \backslash T_{1}\right) \cup\left(B_{2} \backslash T_{2}\right)$ has $f(v) \geqslant n$ and $\Delta(H)-|A|=n-1$, it is easy to greedily $C$-color all vertices of $H-A$. For example, greedily color all vertices of $T_{2}$, followed by those of $B_{2} \backslash T_{2}$, followed by those of $T_{1}$, followed by those of $B_{1} \backslash\left(T_{1} \cup A\right)$. This greedy coloring is possible because at the time we color each vertex it has more available colors than colored neighbors.

We generally follow this approach. However, we modify it so that after we color $H-A$ each vertex in $A$ still has $|A|$ available colors, and we can extend the coloring to $A$. To do this, for each vertex $v \in A$ we will repeatedly "save a color", before greedily coloring the other vertices. To accomplish this we pick vertices $w \in N(v) \cap B_{2}$ and $x \in B_{1} \backslash N(w)$. Now we color $w$ and $x$ with some colors $\alpha$ and $\beta$ (possibly with $\alpha=\beta$ ) such that $\alpha$ and $\beta$ forbid the same color on $v$. For each $v \in A$, we must save a color $\left|N(v) \cap B_{2}\right|$ times. After doing so, we color the remaining vertices greedily (as in the previous paragraph), ending with the vertices of $A$. The only change is that we must ensure that each of the final 11 vertices we color in $B_{2}$ has no colored neighbor in $B_{1}$. In the process of saving colors for vertices in $A$, we color at most $11^{2}$ vertices in $B_{1}$. Each of these forbids at most 11 vertices in $B_{2}$ from appearing among the final 11 in $B_{2}$, for a total of at most $11^{3}$ vertices in $B_{2}$ forbidden. Similarly, we color at most $11^{2}$ 
vertices in $B_{2}$, and these are obviously forbidden from appearing among the final 11 vertices in $B_{2}$. Thus, we can choose the desired 11 final vertices in $B_{2}$ (after saving colors for the vertices in $A$ ), since $\left|B_{2}\right| \geqslant\left|T_{2}\right|+11^{3}+11^{2}+11$.

Note that, while saving colors for some vertex $v \in A$, we color all neighbors of $v$ in $B_{2}$. As a result, we need that no two vertices in $A$ have a common neighbor in $B_{2}$. Each vertex $v \in A$ has at most 11 neighbors in $B_{2}$, and each of these neighbors has at most 10 other neighbors in $B_{1}$. Thus, each $v \in A$ forbids at most 11(10) other vertices from $A$. So, to pick the desired $A$, we need $\left|B_{1}\right|>\left|T_{1}\right|+10(110+1)$.

Now, for each $v \in A$, we repeat the following $\left|N(v) \cap B_{2}\right|$ times. Choose uncolored vertices $w \in N(v) \cap B_{2}$ and $x \in B_{1} \backslash N(w)$. Note that if $N(v) \subset B_{1}$, there is nothing to do at all, hence we may assume that the vertex $w$ exists. Let $g(v), g(w)$, and $g(x)$ denote the number of remaining available colors for $v, w$, and $x$.

$g(v), g(w)$ $g(x)$

Without loss of generality, we assume that the bounds of Hypotheses 1. and 2 . are tight, so that $f(y)=n-44$ for all $y \in T_{1} \cup T_{2}$, and $f(y)=n$ otherwise. Since $A \cap T_{1}=\varnothing$, we have $f(v)=n \geqslant f(w)$, hence we may assume that $C_{v w}$ saturates $\{w\} \times\{1, \cdots, f(w)\}$ (otherwise, add arbitrary edges until this is the case). Thus, each color available for $w$ forbids a color for $v$; similarly for colors available for $x$. By Pigeonhole, if $g(w)+g(x)>n$, then there exist colors $\alpha$ and $\beta$, available for $w$ and $x$ respectively, that both forbid the same color on $v$. Suppose that thus far we have saved a total of $i$ colors for vertices in $A$. Therefore, the $i$ colored vertices of $B_{2}$ forbid $i$ colors for $w$, and its neighbors in $B_{1}$ forbid at most 11 colors, so that we have $g(w) \geqslant f(w)-i-11 \geqslant n-i-11 \geqslant n-131$ and, similarly, $g(x) \geqslant n-131$. We can assume that $g(v) \leqslant f(v) \leqslant n$. And clearly $2(n-131)>n$. Thus, the desired colors $\alpha$ and $\beta$ exist.

It is worth noting that the $\Delta_{0}$ given by our proof of Theorem 3.19 , namely $2642900^{2}$, is much smaller than that arising from our proof of Theorem 3.1, namely $23769500^{2}$. By adapting the statement and proof of Lemma 3.21, we can extend the main result in [3] to correspondence coloring (while also modestly decreasing the $\Delta_{0}$ arising from that proof).

\section{Acknowledgments}

Thanks to four referees for their feedback; in particular, one wrote a very thorough and useful report. Thanks also to Zdeněk Dvořák and Jean-Sébastien Sereni for their helpful comments after carefully reading Section 3. Zdeněk caught a few errors in an earlier version of this paper.

\section{References}

[1] Noga Alon and Michael Tarsi. Colorings and orientations of graphs. Combinatorica, 12(2):125-134, 1992. 
[2] Omid Amini, Louis Esperet, and Jan van den Heuvel. A unified approach to distance-two colouring of graphs on surfaces. Combinatorica, 33:253-296, 2013. Preprint available at https://arxiv.org/abs/0812.1345.

[3] Marthe Bonamy, Daniel W. Cranston, and Luke Postle. Planar graphs of girth at least five are square $(\Delta+2)$-choosable. J. Combin. Theory Ser. B, 134:218-238, 2019. Preprint available at: https://arxiv.org/abs/1508.03663.

[4] Oleg V. Borodin, Aleksei N. Glebov, Anna O. Ivanova, T. K. Neustroeva, and Vladimir A. Tashkinov. Sufficient conditions for planar graphs to be 2-distance $(\Delta+1)$-colorable. Sib. Elektron. Mat. Izv., 1:129-141, 2004.

[5] Daniel W. Cranston and Bobby Jaeger. List-coloring the squares of planar graphs without 4-cycles and 5-cycles. J. Graph Theory, 85(4):721-737, 2017. Preprint available at https://arxiv.org/abs/1505.03197.

[6] Daniel W. Cranston and Douglas B. West. An introduction to the discharging method via graph coloring. Discrete Math., 340(4):766-793, 2017. Preprint available at https://arxiv.org/abs/1306.4434.

[7] Wei Dong and Baogang Xu. 2-distance coloring of planar graphs without 4-cycles and 5-cycles. SIAM J. Discrete Math., To appear.

[8] Zdeněk Dvořák, Daniel Král, Pavel Nejedlý, and Riste Škrekovski. Coloring squares of planar graphs with girth six. European J. Combin., 29(4):838-849, 2008 .

[9] Frédéric Havet, Jan van den Heuvel, Colin McDiarmid, and Bruce Reed. List Colouring Squares of Planar Graphs. July 2008. Preprint available at https: //arxiv.org/abs/0807.3233.

[10] Theodore Kimball Jonas. Graph coloring analogues with a condition at distance two: L(2,1)-labellings and list lambda-labellings. ProQuest LLC, Ann Arbor, MI, 1993. Thesis (Ph.D.)-University of South Carolina.

[11] Michael Molloy and Mohammad R. Salavatipour. A bound on the chromatic number of the square of a planar graph. J. Combin. Theory Ser. B, 94(2):189$213,2005$.

[12] Uwe Schauz. Flexible color lists in Alon and Tarsi's theorem, and time scheduling with unreliable participants. Electronic J. Combin., 17(1):13, 2010.

[13] Wei-Fan Wang and Ko-Wei Lih. Labeling planar graphs with conditions on girth and distance two. SIAM J. Discrete Math., 17(2):264-275, 2003. 